\title{
Accurate identification of fastidious Gram-negative rods: integration of both conventional phenotypic methods and 16S rRNA gene analysis
}

\author{
Maria G de Melo Oliveira ${ }^{1,2}$, Susanne Abels ${ }^{1,3}$, Reinhard Zbinden' ${ }^{1}$ Guido V Bloemberg ${ }^{1}$ and Andrea Zbinden ${ }^{\text {** }}$
}

\begin{abstract}
Background: Accurate identification of fastidious Gram-negative rods (GNR) by conventional phenotypic characteristics is a challenge for diagnostic microbiology. The aim of this study was to evaluate the use of molecular methods, e.g., $16 \mathrm{~S}$ rRNA gene sequence analysis for identification of fastidious GNR in the clinical microbiology laboratory.
\end{abstract}

Results: A total of 158 clinical isolates covering 20 genera and 50 species isolated from 1993 to 2010 were analyzed by comparing biochemical and $16 \mathrm{~S}$ rRNA gene sequence analysis based identification. 16S rRNA gene homology analysis identified 148/158 (94\%) of the isolates to species level, 9/158 (5\%) to genus and 1/158 (1\%) to family level. Compared to 165 rRNA gene sequencing as reference method, phenotypic identification correctly identified 64/158 (40\%) isolates to species level, mainly Aggregatibacter aphrophilus, Cardiobacterium hominis, Eikenella corrodens, Pasteurella multocida, and 21/158 (13\%) isolates correctly to genus level, notably Capnocytophaga sp.; 73/158 (47\%) of the isolates were not identified or misidentified.

Conclusions: We herein propose an efficient strategy for accurate identification of fastidious GNR in the clinical microbiology laboratory by integrating both conventional phenotypic methods and 16S rRNA gene sequence analysis. We conclude that $16 \mathrm{~S}$ rRNA gene sequencing is an effective means for identification of fastidious GNR, which are not readily identified by conventional phenotypic methods.

Keywords: Fastidious Gram-negative rods, 165 rRNA gene, Conventional phenotypic methods

\section{Background}

Accurate identification of fastidious Gram-negative rods (GNR) is a challenge for clinical microbiology laboratories. Fastidious GNR are slow-growing organisms, which generally require supplemented media or $\mathrm{CO}_{2}$ enriched atmosphere and fail to grow on enteric media such as MacConkey agar [1]. They are isolated infrequently and consist of different taxa including Actinobacillus, Capnocytophaga, Cardiobacterium, Eikenella, Kingella, Moraxella, Neisseria, and Pasteurella. Most of them are colonizers of the human oral cavity but they have been demonstrated to cause severe systemic infections like endocarditis, septicemia and abscesses, particularly in immunocompromised patients $[1,2]$. Accurate identification of fastidious GNR is of concern

\footnotetext{
* Correspondence: azbinden@imm.uzh.ch

${ }^{1}$ Institute of Medical Microbiology, University of Zurich, 8006 Zurich,

Switzerland

Full list of author information is available at the end of the article
}

when isolated from normally sterile body sites regarding guidance of appropriate antimicrobial therapy and patient management [1].

Identification of fastidious GNR by conventional methods is difficult and time-consuming because phenotypic characteristics such as growth factor requirements, fermentation and assimilation of carbohydrates, morphology, and staining behaviour are subject to variation and dependent on individual interpretation and expertise $[1,3]$. Commercially available identification systems such as VITEK 2 NH (bioMérieux, Marcy L'Etoile, France) only partially allow for accurate identification of this group of microorganisms, e.g., Eikenella corrodens, Kingella kingae and Cardiobacterium hominis [4-6]. Most studies relied only on a subset of taxa of fastidious GNR or did not include clinical isolates under routine conditions [4-6]. The application of newer identification methods like matrix-assisted laser desorption ionization-

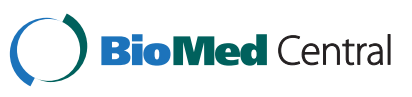


time of flight mass spectrometry (MALDI-TOF MS) shows promising results regarding the identification of HACEK group members (Haemophilus parainfluenzae, Aggregatibacter spp., Cardiobacterium spp., E. corrodens, and Kingella spp.), however, only a small set of isolates and species were investigated [7-9]. Other potentially pathogenic fastidious GNR such as Capnocytophaga spp. or Pasteurella spp., which are known agents of wound infections and septicemia after animal bites [1] frequently are not included in comparative analyses. In addition, implementation of MALDI-TOF identification also depends on the number of correctly identified reference strains in the database.

$16 \mathrm{~S}$ rRNA gene sequence analysis is generally considered as the "gold standard" for bacterial identification [3,10,11]. We analysed a substantial data set of 158 clinical fastidious GNR isolates covering various difficult-to-identify taxa, which were collected during a 17-year period. We propose a feasible strategy for accurate identification of fastidious GNR in a routine diagnostic laboratory using both conventional phenotypic and molecular methods, e.g., 16S rRNA gene analysis.

\section{Methods}

\section{Clinical isolates}

The 158 isolates of fastidious GNR included in this study derived from clinical human specimens taken as part of standard patient care and were collected from 1993 to 2010 at the Institute of Medical Microbiology, University of Zurich, Switzerland. All isolates were identified both by conventional biochemical methods and $16 \mathrm{~S}$ rRNA gene sequence analysis. The isolates were cultured on Columbia sheep blood or chocolate agar (Becton, Dickinson \& Company, Franklin Lakes, NJ (BD)) and incubated at $37^{\circ} \mathrm{C}$ with $5 \% \mathrm{CO}_{2}$ for 24 to $48 \mathrm{~h}$. The isolates were stored at $-80^{\circ} \mathrm{C}$ as pure cultures.

\section{Biochemical identification}

The isolates were identified using in-house biochemical reactions as described for coryneform bacteria, for unusual Gram-negative aerobic bacteria and for facultative anaerobic bacteria $[12,13]$. In addition to the Gram stain, the following biochemical reactions were investigated: catalase, oxidase, nitrate reduction, urease, indole production, ornithine decarboxylase, hydrolysis of esculin; acid production from glucose, sucrose, maltose, mannitol and xylose was tested in semisolid cystine-trypticase agar medium (BD) supplemented with rabbit serum; tests for fermentative/nonfermentative carbohydrate metabolism were done on triple sugar iron agar. Identification by biochemical methods was scored as correct or incorrect taxonomic level compared to the $16 \mathrm{~S}$ rRNA gene analysis as reference method. An incorrect assignment to species level was scored as incorrect species even if the genus was correct. If biochemical identification methods did not assign an isolate to at least genus level, the strain was scored as not identified.

\section{S rRNA gene sequence analysis}

Sequencing of the partial $16 \mathrm{~S}$ rRNA gene was performed as described previously [14]. In brief, a loopful of bacterial cells was used for extraction of DNA by lysozyme digestion and alkaline hydrolysis. Nucleic acids were purified using the QIAamp DNA blood kit (Qiagen AG, Basel, Switzerland). The 5'-part of the $16 \mathrm{~S}$ rRNA gene (corresponding to Escherichia coli positions 10 to 806) was amplified using primers BAK11w [5'-AGTTTGATC (A/C)TGGCTCAG] and BAK2 [5'-GGACTAC(C/T/A) AGGGTATCTAAT]. Amplicons were purified and sequenced with forward primer BAK11w using an automatic DNA sequencer (ABI Prism 310 Genetic Analyzer; Applied Biosystems, Rotkreuz, Switzerland).

BLAST search of partial 16S rRNA gene sequences was performed by using Smartgene database (SmartGene ${ }^{\mathrm{TM}}$, Zug, Switzerland) on March 2013. The SmartGene database is updated with the newest $16 \mathrm{~S}$ rRNA gene sequences from NCBI GenBank through an automated process every day. Non-validated taxa or non published sequences were not taken into consideration. The following criteria were used for 16S rRNA gene based identification [14-17]: (i) when the comparison of the sequence determined with a sequence in the database of a classified species yielded a similarity score of $\geq 99 \%$, the isolate was assigned to that species; (ii) when the score was $<99 \%$ and $\geq 95 \%$, the isolate was assigned to the corresponding genus; (iii) when the score was $<95 \%$, the isolate was assigned to a family. If the unknown isolate was assigned to a species and the second classified species in the scoring list showed less than $0.5 \%$ additional sequence divergence, the isolate was categorized as identified to the species level but with low demarcation. The sequence analysis was considered as the reference method but in cases with low demarcation results of supplemental conventional tests were taken into consideration for the final identification. Partial 16S rRNA gene sequences of all 158 clinical isolates were deposited in NCBI GenBank under GenBank accession numbers KC866143-KC866299 and GU797849, respectively.

\section{VITEK 2 NH card identification}

A subset of 80 of the total of 158 isolates was tested by the colorimetric VITEK $2 \mathrm{NH}$ card (bioMérieux) according to the instructions of the manufacturer. The colorimetric VITEK $2 \mathrm{NH}$ card contains 30 tests and the corresponding database covers 26 taxa. Identification by VITEK $2 \mathrm{NH}$ was compared to the $16 \mathrm{~S}$ rRNA gene analysis as reference method. 
Table 1 Identification of clinical isolates $(n=158)$ by conventional methods compared to $16 S$ rRNA gene sequence analysis

\begin{tabular}{|c|c|c|c|c|c|}
\hline \multicolumn{2}{|l|}{ Conventional phenotyic methods } & \multicolumn{3}{|l|}{$\begin{array}{l}\text { 16S rRNA gene sequence } \\
\text { analysis }\end{array}$} & \multirow[t]{2}{*}{$\begin{array}{l}\text { Final identification (supplemental } \\
\text { conventional tests if required) }\end{array}$} \\
\hline Identification (number of isolates) & $\begin{array}{l}\text { Level of } \\
\text { identification } \\
\text { and correctness } \\
\text { of result }\end{array}$ & $\begin{array}{l}\text { Best reference species } \\
\text { sequence }\end{array}$ & $\begin{array}{l}\% \text { difference to } \\
\text { reference species } \\
\text { sequence }\end{array}$ & $\begin{array}{l}\text { GenBank accession } \\
\text { numbers }\end{array}$ & \\
\hline Actinobacillus ureae (1) & $\mathrm{S}^{1} ; \mathrm{SI}^{2}$ & $\begin{array}{l}\text { Actinobacillus hominis } \\
\text { Actinobacillus suis (low } \\
\text { demarcation) }\end{array}$ & $0.0,0.4$ & KC866152 & $\begin{array}{l}\text { A. hominis (acidification of mannitol: } \\
\text { A. hominis (positive), A. suis } \\
\text { (negative) [1]) }\end{array}$ \\
\hline $\begin{array}{l}\text { Aggregatibacter } \\
\text { actinomycetemcomitans (2) }\end{array}$ & S; SC & $\begin{array}{l}\text { Aggregatibacter } \\
\text { actinomycetemcomitans }\end{array}$ & $0.0,0.3$ & KC866227; KC866228 & A. actinomycetemcomitans \\
\hline $\begin{array}{l}\text { Aggregatibacter } \\
\text { actinomycetemcomitans (1) }\end{array}$ & S; Sl & Pasteurella bettyae & 0.0 & KC866143 & P. bettyae \\
\hline Aggregatibacter aphrophilus (11) & S; SC & Aggregatibacter aphrophilus & $0.0-0.8$ & $\begin{array}{l}\text { KC866144; KC866145; } \\
\text { KC866146; KC866147; } \\
\text { KC866148; KC866149; } \\
\text { KC866150; KC866229; } \\
\text { KC866230; KC866231; } \\
\text { KC866272 }\end{array}$ & A. aphrophilus \\
\hline Aggregatibacter aphrophilus (2) & $\mathrm{S} ; \mathrm{Sl}$ & Aggregatibacter aphrophilus & $3.8,2.9$ & KC866151; KC866153 & Aggregatibacter sp. \\
\hline \multirow[t]{3}{*}{ Aggregatibacter aphrophilus (1) } & \multirow[t]{3}{*}{$\mathrm{S} ; \mathrm{Sl}$} & Neisseria sicca & 0.8 & \multirow[t]{3}{*}{ KC866154 } & \multirow{3}{*}{$\begin{array}{l}\text { N. sicca (nitrate reduction: positive } \\
\text { (N. mucosa), negative (N. sicca, N. } \\
\text { subflava bv. flava); sucrose } \\
\text { acidification: positive (N. sicca, N. } \\
\text { mucosa), negative (N. subflava bv. } \\
\text { flava) [18]) }\end{array}$} \\
\hline & & Neisseria subflava bv. flava & 1.0 & & \\
\hline & & $\begin{array}{l}\text { Neisseria mucosa (low } \\
\text { demarcation) }\end{array}$ & 1.1 & & \\
\hline Aggregatibacter sp. (1) & $\mathrm{G} ; \mathrm{GC}$ & Aggregatibacter aphrophilus & 2.3 & KC866155 & Aggregatibacter sp. \\
\hline Bergeyella zoohelcum (1) & $\mathrm{S} ; \mathrm{Sl}$ & Myroides odoratimimus & 5.9 & KC866156 & Flavobacteriaceae \\
\hline Bergeyella zoohelcum (1) & $\mathrm{S} ; \mathrm{Sl}$ & Neisseria zoodegmatis & 0.3 & KC866157 & N. zoodegmatis \\
\hline Capnocytophaga canimorsus (2) & $\mathrm{S} ; \mathrm{SC}$ & Capnocytophaga canimorsus & $0.5,0.4$ & KC866158; KC866159 & C. canimorsus \\
\hline Capnocytophaga ochracea (1) & S; SI & Capnocytophaga gingivalis & 0.6 & KC866160 & C. gingivalis \\
\hline Capnocytophaga ochracea (1) & $\mathrm{S} ; \mathrm{Sl}$ & Capnocytophaga ochracea & 2.5 & KC866161 & Capnocytophaga sp. \\
\hline Capnocytophaga ochracea (5) & $\mathrm{S} ; \mathrm{Sl}$ & Capnocytophaga sputigena & $0.0-0.3$ & $\begin{array}{l}\text { KC866162; KC866163; } \\
\text { KC866164; KC866273; } \\
\text { KC866274 }\end{array}$ & C. sputigena ${ }^{3}$ \\
\hline Capnocytophaga ochracea (1) & $\mathrm{S} ; \mathrm{Sl}$ & Dysgonomonas mossii & 0.6 & KC866165 & D. mossii \\
\hline Capnocytophaga ochracea (1) & $\mathrm{S} ; \mathrm{Sl}$ & Leptotrichia trevisanii & 0.2 & KC866166 & L. trevisanii \\
\hline Capnocytophaga sp. (2) & $\mathrm{G} ; \mathrm{GC}$ & Capnocytophaga sputigena & $0.0,0.6$ & KC866167; KC866232 & C. sputigena \\
\hline Cardiobacterium hominis (4) & $\mathrm{S} ; \mathrm{SC}$ & Cardiobacterium hominis & $0.0-0.5$ & $\begin{array}{l}\text { KC866168; KC866233; } \\
\text { KC866275; KC866299 }\end{array}$ & C. hominis \\
\hline
\end{tabular}


Table 1 Identification of clinical isolates $(n=158)$ by conventional methods compared to 16S rRNA gene sequence analysis (Continued)

\begin{tabular}{|c|c|c|c|c|c|}
\hline \multirow[t]{2}{*}{ CDC Group lle (1) } & \multirow[t]{2}{*}{ S; SI } & Chryseobacterium anthropi & 0.2 & \multirow[t]{2}{*}{ KC866169 } & \multirow{2}{*}{$\begin{array}{l}\text { C. anthropi (acidification of fructose } \\
\text { and sucrose: positive (C. haifense), } \\
\text { negative (C. anthropi) [19]) }\end{array}$} \\
\hline & & $\begin{array}{l}\text { Chryseobacterium haifense (low } \\
\text { demarcation) }\end{array}$ & 0.6 & & \\
\hline Comamonas sp. (1) & $\mathrm{G} ; \mathrm{Gl}$ & Oligella urethralis & 0.0 & KC866170 & O. urethralis \\
\hline Dysgonomonas capnocytophagoides (1) & S; SC & $\begin{array}{l}\text { Dysgonomonas } \\
\text { capnocytophagoides }\end{array}$ & 0.2 & KC866171 & D. capnocytophagoides \\
\hline Eikenella corrodens (10) & $\mathrm{S} ; \mathrm{SC}$ & Eikenella corrodens & $0.0-0.8$ & $\begin{array}{l}\text { KC866172; KC866173; } \\
\text { KC866174; KC866175; } \\
\text { KC866176; KC866177; } \\
\text { KC866178; KC866234; } \\
\text { KC866235; KC866236 }\end{array}$ & E. corrodens \\
\hline Flavobacterium sp. (1) & G; GC & Flavobacterium lindanitolerans & 0.4 & KC866179 & F. lindanitolerans \\
\hline Gram-negative rods (1) & $\mathrm{N}$ & Actinobacillus hominis & 0.3 & KC866238 & A. hominis \\
\hline \multirow[t]{3}{*}{ Gram-negative rods (1) } & \multirow[t]{3}{*}{ N } & Actinobacillus hominis & 0.0 & \multirow[t]{3}{*}{ KC866237 } & \multirow{3}{*}{$\begin{array}{l}\text { A. hominis (esculin hydrolysis: } \\
\text { positive (A. suis), variable (A. hominis), } \\
\text { negative (A. equuli); mannitol } \\
\text { acidification: positive (A. equuli, A. } \\
\text { hominis), negative (A. suis) [1]) }\end{array}$} \\
\hline & & Actinobacillus suis & 0.0 & & \\
\hline & & $\begin{array}{l}\text { Actinobacillus equuli (low } \\
\text { demarcation) }\end{array}$ & 0.5 & & \\
\hline Gram-negative rods (1) & $\mathrm{N}$ & $\begin{array}{l}\text { Aggregatibacter } \\
\text { actinomycetemcomitans }\end{array}$ & 0.2 & KC866239 & A. actinomycetemcomitans \\
\hline Gram-negative rods (2) & N & Aggregatibacter aphrophilus & $0.3,0.8$ & KC866240; KC866241 & A. aphrophilus \\
\hline Gram-negative rods (1) & N & Azospira oryzae & 0.0 & KC866276 & A. oryzae \\
\hline Gram-negative rods (1) & $\mathrm{N}$ & Brevundimonas terrae & 0.6 & KC866180 & B. terrae \\
\hline Gram-negative rods (3) & $\mathrm{N}$ & Capnocytophaga canimorsus & $0.0-0.2$ & $\begin{array}{l}\text { KC866277; KC866278; } \\
\text { KC866279 }\end{array}$ & C. canimorsus \\
\hline Gram-negative rods (1) & $\mathrm{N}$ & Capnocytophaga sputigena & 0.0 & KC866280 & C. sputigena \\
\hline Gram-negative rods (2) & $\mathrm{N}$ & Cardiobacterium hominis & $0.5,0.6$ & KC866281; KC866282 & C. hominis \\
\hline \multirow[t]{2}{*}{ Gram-negative rods (1) } & \multirow[t]{2}{*}{$\mathrm{N}$} & Chryseobacterium haifense & 0.2 & \multirow[t]{2}{*}{ KC866181 } & \multirow{2}{*}{$\begin{array}{l}\text { C. anthropi (acidification of fructose } \\
\text { and sucrose: positive (C. haifense), } \\
\text { negative (C. anthropi) [19]) }\end{array}$} \\
\hline & & $\begin{array}{l}\text { Chryseobacterium anthropi (low } \\
\text { demarcation) }\end{array}$ & 0.5 & & \\
\hline Gram-negative rods (1) & N & Kingella denitrificans & 0.0 & KC866182 & K. denitrificans \\
\hline Gram-negative rods (1) & N & Moraxella atlantae & 0.2 & KC866242 & M. atlantae \\
\hline Gram-negative rods (2) & $\mathrm{N}$ & Moraxella lacunata & 0.0 & KC866283; KC866284 & M. lacunata \\
\hline Gram-negative rods (1) & $\mathrm{N}$ & Moraxella lincolnii & 0.3 & KC866243 & M. lincolnii \\
\hline Gram-negative rods (3) & N & Moraxella nonliquefaciens & $0.0-0.7$ & $\begin{array}{l}\text { KC866285; KC866286; } \\
\text { KC866287 }\end{array}$ & M. nonliquefaciens \\
\hline Gram-negative rods (2) & $\mathrm{N}$ & Moraxella osloensis & $0.0,0.2$ & KC866288; KC866289 & M. osloensis \\
\hline Gram-negative rods (1) & $\mathrm{N}$ & Neisseria bacilliformis & 0.0 & KC866244 & N. bacilliformis \\
\hline
\end{tabular}

Actinobacillus suis

positive (A. suis), variable (A. hominis),

(A. equuli); mannitol

(A. equuli, A.

demarcation)

KC866239

actinomycetemcomitans

Aggregatibacter aphrophilus

\section{KC866276}

A. aphrophilus

Brevundimonas terrae

KC866277; KC866278;

apnocytophaga canimorsus

KC866280

Cardiobacterium hominis

KC866281; KC866282

C. hominis

Chryseobacterium anthropi (low

C. anthropi (acidification of fructose

K. denitrifican

M. atlantae

Moraxella lincolnir

N. bacilliformis 
Table 1 Identification of clinical isolates $(n=158)$ by conventional methods compared to $16 \mathrm{~S}$ rRNA gene sequence analysis (Continued)

\begin{tabular}{|c|c|c|c|c|c|}
\hline Gram-negative rods (1) & $\mathrm{N}$ & Neisseria zoodegmatis & 2.0 & KC866245 & Neisseria sp. \\
\hline Gram-negative rods (4) & $\mathrm{N}$ & Neisseria elongata & $0.0-0.3$ & $\begin{array}{l}\text { KC866246; KC866247; } \\
\text { KC866290; KC866291 }\end{array}$ & N. elongata \\
\hline \multirow[t]{2}{*}{ Gram-negative rods (1) } & \multirow[t]{2}{*}{$\mathrm{N}$} & Neisseria flavescens & 0.5 & \multirow[t]{2}{*}{ KC866248 } & \multirow{2}{*}{$\begin{array}{l}\text { N. subflava (acidification of glucose } \\
\text { and maltose: positive ( } N \text {. subflava), } \\
\text { negative ( } N \text {. flavescens) [18]) }\end{array}$} \\
\hline & & $\begin{array}{l}\text { Neisseria subflava (low } \\
\text { demarcation) }\end{array}$ & 0.7 & & \\
\hline \multirow[t]{2}{*}{ Gram-negative rods (2) } & \multirow[t]{2}{*}{$\mathrm{N}$} & Neisseria flavescens & 0.3 & \multirow[t]{2}{*}{ KC866249; KC866250 } & \multirow{2}{*}{$\begin{array}{l}\text { N. subflava (acidification of glucose } \\
\text { and maltose: positive (N. subflava), } \\
\text { negative (N. flavescens) [18]) }\end{array}$} \\
\hline & & $\begin{array}{l}\text { Neisseria subflava (low } \\
\text { demarcation) }\end{array}$ & 0.4 & & \\
\hline Gram-negative rods (4) & $\mathrm{N}$ & Neisseria weaveri & $0.0-0.3$ & $\begin{array}{l}\text { KC866251; KC866252; } \\
\text { KC866253; KC866254 }\end{array}$ & N. weaveri \\
\hline Gram-negative rods (1) & $\mathrm{N}$ & Pasteurella bettyae & 0.0 & KC866292 & P. bettyae \\
\hline \multirow[t]{2}{*}{ Gram-negative rods (1) } & \multirow[t]{2}{*}{$\mathrm{N}$} & Pasteurella dagmatis & 0.4 & \multirow[t]{2}{*}{ KC866255 } & \multirow{2}{*}{$\begin{array}{l}\text { P. stomatis (urease reaction: positive } \\
\text { (P. dagmatis), negative ( } P \text {. stomatis); } \\
\text { acidification of maltose: positive }(P \text {. } \\
\text { dagmatis), negative }(P \text {. stomatis) [1]) }\end{array}$} \\
\hline & & $\begin{array}{l}\text { Pasteurella stomatis (low } \\
\text { demarcation) }\end{array}$ & 0.4 & & \\
\hline Kingella denitrificans (1) & S; SC & Kingella denitrificans & 0.6 & KC866183 & K. denitrificans \\
\hline Kingella denitrificans (1) & S; SI & Neisseria elongata & 0.0 & KC866184 & N. elongata \\
\hline Leptotrichia buccalis (1) & $\mathrm{S} ; \mathrm{SI}$ & Leptotrichia trevisanii & 0.3 & KC866293 & L. trevisanii \\
\hline \multirow[t]{2}{*}{ Moraxella lacunata (1) } & \multirow[t]{2}{*}{$\mathrm{S} ; \mathrm{SC}$} & Moraxella lacunata & 0.5 & \multirow[t]{2}{*}{ KC866185 } & \multirow{2}{*}{$\begin{array}{l}\text { M. lacunata (gelatinase reaction: } \\
\text { positive (M. lacunata), negative ( } M \text {. } \\
\text { nonliquefaciens) [20]) }\end{array}$} \\
\hline & & $\begin{array}{l}\text { Moraxella nonliquefaciens (low } \\
\text { demarcation) }\end{array}$ & 0.7 & & \\
\hline Moraxella osloensis (1) & S; SC & Moraxella osloensis & 0.0 & KC866186 & M. osloensis \\
\hline \multirow[t]{2}{*}{ Moraxella osloensis (1) } & \multirow[t]{2}{*}{$\mathrm{S} ; \mathrm{Sl}$} & Psychrobacter faecalis & 0.0 & \multirow[t]{2}{*}{ KC866187 } & \multirow{2}{*}{$\begin{array}{l}\text { P. pulmonis (acidification of glucose } \\
\text { and xylose: positive (P. faecalis), } \\
\text { negative (P. pulmonis) [20]) }\end{array}$} \\
\hline & & $\begin{array}{l}\text { Psychrobacter pulmonis (low } \\
\text { demarcation) }\end{array}$ & 0.2 & & \\
\hline Moraxella sp. (1) & $\mathrm{G} ; \mathrm{GC}$ & Moraxella canis & 0.2 & KC866188 & M. canis \\
\hline Neisseria sp. (1) & $\mathrm{G} ; \mathrm{Gl}$ & Neisseria elongata & 0.3 & KC866256 & N. elongata \\
\hline Moraxella sp. (4) & $\mathrm{G} ; \mathrm{GC}$ & Moraxella nonliquefaciens & $0.0-0.3$ & $\begin{array}{l}\text { KC866189; KC866190; } \\
\text { KC866257; KC866258 }\end{array}$ & M. nonliquefaciens \\
\hline Moraxella sp. (8) & $\mathrm{G} ; \mathrm{GC}$ & Moraxella osloensis & $0.0-0.2$ & $\begin{array}{l}\text { KC866191; KC866192; } \\
\text { KC866193; KC866194; } \\
\text { KC866259; KC866260; } \\
\text { KC866261; KC866294 }\end{array}$ & M. osloensis \\
\hline Neisseria animaloris (EF4a) (1) & $\mathrm{S} ; \mathrm{SC}$ & Neisseria animaloris & 0.0 & KC866195 & N. animaloris \\
\hline Neisseria animaloris (EF4a) (1) & $\mathrm{S} ; \mathrm{SI}$ & Neisseria zoodegmatis & 0.0 & GU797849 & N. zoodegmatis \\
\hline Neisseria cinerea (2) & S; SC & Neisseria cinerea & 0.0 & KC866196; KC866197 & $\begin{array}{l}\text { N. cinerea (acidification of glucose } \\
\text { and maltose: positive ( } N \text {. }\end{array}$ \\
\hline
\end{tabular}


Table 1 Identification of clinical isolates $(n=158)$ by conventional methods compared to $16 \mathrm{~S}$ rRNA gene sequence analysis (Continued)

\begin{tabular}{|c|c|c|c|c|c|}
\hline & & $\begin{array}{l}\text { Neisseria meningitidis (low } \\
\text { demarcation) }\end{array}$ & 0.3 & & $\begin{array}{l}\text { meningitidis), negative ( } N \text {. cinerea) } \\
{[18] \text { ) }}\end{array}$ \\
\hline Neisseria elongata (1) & S; SI & Aggregatibacter aphrophilus & 2.4 & KC866198 & Aggregatibacter sp. \\
\hline Neisseria elongata (3) & S; SC & Neisseria elongata & $0.0-0.3$ & $\begin{array}{l}\text { KC866203; KC866204; } \\
\text { KC866205 }\end{array}$ & N. elongata \\
\hline Neisseria elongata (2) & S; SI & Neisseria bacilliformis & $0.1,0.4$ & KC866201; KC866202 & N. bacilliformis \\
\hline Neisseria elongata (1) & S; SI & Neisseria zoodegmatis & 0.6 & KC866206 & N. zoodegmatis \\
\hline Neisseria elongata (2) & S; SI & Eikenella corrodens & 0.0 & KC866199; KC866200 & E. corrodens \\
\hline Neisseria sp. (1) & $\mathrm{G} ; \mathrm{GC}$ & Neisseria shayeganii & 0.3 & KC866207 & N. shayeganii \\
\hline Neisseria sp. (1) & $\mathrm{G} ; \mathrm{GC}$ & Neisseria elongata & 0.2 & KC866270 & N. elongata \\
\hline Neisseria sp. (1) & $\mathrm{G} ; \mathrm{GC}$ & Neisseria oralis & 0.0 & KC866208 & N. oralis \\
\hline Neisseria weaveri (1) & S; SC & Neisseria weaveri & 0.0 & KC866211 & N. weaveri \\
\hline Neisseria weaveri (1) & S; SC & Neisseria shayeganii & 0.2 & KC866210 & N. shayeganii \\
\hline Neisseria weaveri (1) & S; SI & Azospira oryzae & 0.0 & KC866209 & A. oryzae \\
\hline Neisseria zoodegmatis (EF4b) (3) & S; SC & Neisseria zoodegmatis & $0.0-0.5$ & $\begin{array}{l}\text { KC866212; KC866213; } \\
\text { KC866295 }\end{array}$ & N. zoodegmatis \\
\hline Oligella urethralis (2) & S; SC & Oligella urethralis & 0.0 & KC866214; KC866215 & O. urethralis \\
\hline Pasteurella aerogenes (1) & S; SI & Pasteurella aerogenes & 2.7 & KC866226 & Pasteurella sp. \\
\hline Pasteurella bettyae (2) & S; SC & Pasteurella bettyae & 0.0 & KC866216; KC866262 & P. bettyae \\
\hline Pasteurella canis (1) & S; SC & Pasteurella canis & 0.0 & KC866217 & P. canis \\
\hline Pasteurella canis (1) & S; SI & Pasteurella stomatis & 1.6 & KC866218 & Pasteurella sp. \\
\hline Pasteurella dagmatis (1) & S; SC & Pasteurella dagmatis & 0.2 & KC866271 & P. dagmatis \\
\hline Pasteurella multocida (14) & S; SC & Pasteurella multocida & $0.0-0.2$ & $\begin{array}{l}\text { KC866219; KC866220; } \\
\text { KC866221; KC866222; } \\
\text { KC866223; KC866263; } \\
\text { KC866264; KC866265; } \\
\text { KC866266; KC866267; } \\
\text { KC866268; KC866296; } \\
\text { KC866297; KC866298 }\end{array}$ & P. multocida \\
\hline Pasteurella pneumotropica (1) & S; SI & Bisgaard Taxon 22 & 1.7 & KC866224 & Pasteurella sp. \\
\hline Pasteurella sp. (1) & $\mathrm{G} ; \mathrm{Gl}$ & Necropsobacter rosorum & 0.0 & KC866269 & N. rosorum \\
\hline Roseomonas sp. (1) & $\mathrm{G} ; \mathrm{GC}$ & Roseomonas mucosa & 0.0 & KC866225 & R. mucosa \\
\hline
\end{tabular}

Assignment to taxonomic level: $\mathrm{S}=$ species, $\mathrm{G}=$ genus, $\mathrm{N}=$ not identified.

${ }^{2}$ Correctness of assignment: $\mathrm{SC}=$ correct at species level, $\mathrm{SI}=$ incorrect at species level, $\mathrm{GC}=$ correct at genus level, $\mathrm{Gl}=$ incorrect at genus level, $\mathrm{N}=$ not identified.

Difficult differentiation of species in question by conventional tests. 
Table 2 Summary of identification of fastidious GNR isolates $(n=158)$

\begin{tabular}{|c|c|c|c|c|c|}
\hline \multirow[t]{2}{*}{ Identification procedure } & \multicolumn{2}{|c|}{$\%$ correct identification at taxonomic level } & \multicolumn{3}{|c|}{$\%$ incorrect assignment at taxonomic level or no identification } \\
\hline & Species & Genus & Species & Genus & No identification \\
\hline $\begin{array}{l}16 \mathrm{~S} \text { rRNA gene sequence } \\
\text { analysis }\end{array}$ & $94 \%(n=148)$ & $5 \%(n=9)$ & - & - & $1 \%(n=1)$ \\
\hline $\begin{array}{l}\text { Conventional phenotypic } \\
\text { methods }\end{array}$ & $40 \%(n=64)$ & $13 \%(n=21)$ & $20 \%(n=31)$ & $2 \%(n=3)$ & $25 \%(n=39)$ \\
\hline
\end{tabular}

\section{Results}

One hundred fifty-eight clinically relevant human isolates of fastidious GNR (including rod forms of the genus Neisseria) were collected in our diagnostic laboratory during a 17 -year period. Most of the 158 fastidious GNR isolates belonged to the following genera: Neisseria $(\mathrm{n}=35)$, Pasteurella $(\mathrm{n}=25)$, Moraxella $(\mathrm{n}=24)$, Aggregatibacter $(\mathrm{n}=20)$, Capnocytophaga $(\mathrm{n}=15)$, Eikenella $(\mathrm{n}=12)$, Cardiobacterium $(\mathrm{n}=6)$, Actinobacillus $(\mathrm{n}=3)$, Oligella $(\mathrm{n}=3)$, and Kingella $(\mathrm{n}=2)$ (Table 1). 16S rRNA gene analysis identified $94 \%$ of the 158 isolates to species level and 5\% to genus level; one isolate could only be assigned to family level (Tables 1 and 2). Thirteen isolates were assigned to species level with low demarcation to the next species but supplemental conventional tests revealed a final identification to species level (Table 1). Conventional methods assigned $60 \%$ of the isolates to species level and $15 \%$ to genus level (Tables 1 and 2). However, only $40 \%$ were correctly assigned to species level and $13 \%$ correct to genus level considering the 16S rRNA gene sequencing as reference method. $47 \%$ of the isolates were misidentified or not identified by conventional methods; nevertheless, 18 of the 31 isolates incorrectly assigned to species level were identified to the correct genus (Table 2).

Table 3 Taxa with mostly reliable identification of fastidious GNR by conventional phenotypic methods

\begin{tabular}{ll}
\hline $\begin{array}{l}\text { Conventional phenotypic methods } \\
\text { (number of isolates) }\end{array}$ & Final identification ${ }^{\mathbf{1}}$ \\
\hline Aggregatibacter aphrophilus (14) & A. aphrophilus (11) \\
& Aggregatibacter sp. (2) \\
& Neisseria sicca (1) \\
Capnocytophaga canimorsus (2) & C. canimorsus (2) \\
Capnocytophaga sp. (11) & C. sputigena (7) \\
& C. gingivalis (1) \\
& Capnocytophaga sp. (1) \\
& Dysgonomonas mossii (1) \\
Cardiobacterium hominis (4) & Leptotrichia trevisanii (1) \\
Eikenella corrodens (10) & C. hominis (4) \\
Pasteurella multocida (14) & E. corrodens (10) \\
\hline Tina idido (14)
\end{tabular}

${ }^{1}$ Final identification was assigned using $16 \mathrm{~S}$ rRNA gene identification as the reference method and if required with supplemental conventional tests.
Conventional methods mostly misidentified Moraxella spp. and Neisseria spp.; only 2 out of 24 Moraxella spp., 3 out of 10 Neisseria elongata and 1 out of 5 Neisseria weaveri, respectively, were correctly identified to species level. In contrast, results of phenotypic identification of Aggregatibacter aphrophilus, Cardiobacterium hominis, E. corrodens, Pasteurella multocida and Capnocytophaga sp. other than Capnocytophaga canimorsus were largely congruent with 16S rRNA gene sequence analysis (Table 3). These bacteria display biochemical key reactions that differentiate them from other fastidious GNR; e.g., a positive ornithine decarboxylase reaction and missing sugar acidification in the cystine-trypticase agar medium is typical for E. corrodens; a blood culture isolate with a positive indole reaction and a negative catalase is diagnostic for C. hominis; $P$. multocida has a typical pattern of acidification of sugars and a positive indole reaction and together with a history of cat bite the diagnosis is feasible [1]. C. canimorsus differs from Capnocytophaga gingivalis, Capnocytophaga ochracea, Capnocytophaga sputigena by the positive catalase and oxidase - together with the typical morphology of spindle-shaped cells in the Gram stain and the anamnestic history of a dog bite, the identification is possible with conventional methods; the other Capnocytophaga spp. with a negative catalase and oxidase are difficult to differentiate by conventional methods but identification to the genus level is feasible [21].

The 80 out of 158 isolates analysed by the VITEK $2 \mathrm{NH}$ card belonged to the following genera: Neisseria $(\mathrm{n}=21)$, Moraxella $(\mathrm{n}=13)$, Eikenella $(\mathrm{n}=12)$, Aggregatibacter $(\mathrm{n}=11)$, Pasteurella ( $\mathrm{n}=9)$, Capnocytophaga $(\mathrm{n}=6)$, Actinobacillus $(\mathrm{n}=2)$, Cardiobacterium $(\mathrm{n}=2)$, Kingella $(\mathrm{n}=2)$, Dysgonomonas $(\mathrm{n}=1)$ and Leptotrichia $(\mathrm{n}=1)$ (Table 4). The VITEK $2 \mathrm{NH}$ card identified 25 (31\%) and 7 (9\%) isolates to correct species and genus level, respectively; 4 isolates were assigned to incorrect genus and 21 isolates were not identified; 12 of the further 23 isolates incorrectly assigned to species level were identified to correct genus (Table 4). However, the VITEK $2 \mathrm{NH}$ database includes taxa of only 43 of the 80 isolates studied. Regarding only taxa included in the VITEK $2 \mathrm{NH}$ database, 25 (58\%) and 7 (16\%) out of 43 isolates were identified to correct species and genus level, respectively. The VITEK $2 \mathrm{NH}$ card supports the identification of $A$. aphrophilus, C. hominis, E. corrodens, Capnocytophaga sp. and Kingella sp. 
Table 4 Clinical isolates tested by the colorimetric VITEK 2 NH card $(n=80)$

\begin{tabular}{|c|c|c|}
\hline VITEK 2 NH card (number of isolates) & Level of identification and correctness of result & Final identification $^{1}$ \\
\hline Actinobacillus ureae (1) & $\mathrm{S}^{2} ; \mathrm{Sl}^{3}$ & A. hominis \\
\hline Aggregatibacter aphrophilus (5) & S; SC & A. aphrophilus $^{4}$ \\
\hline Aggregatibacter aphrophilus/Haemophilus parainfluenzae ${ }^{5}$ (3) & $\mathrm{G} ; \mathrm{GC}$ & A. aphrophilus ${ }^{4}$ \\
\hline Campylobacter fetus/coli (2) & G; Gl & Moraxella osloensis \\
\hline Capnocytophaga sp. (4) & G; GC & C. sputigena ${ }^{4}$ \\
\hline Capnocytophaga sp. (1) & G; Gl & Dysgonomonas mossii \\
\hline Capnocytophaga sp. (1) & G; Gl & Leptotrichia trevisanii \\
\hline Cardiobacterium hominis (2) & S; SC & C. hominis ${ }^{4}$ \\
\hline Eikenella corrodens (11) & S; SC & E. corrodens ${ }^{4}$ \\
\hline Eikenella corrodens (1) & S; SI & Neisseria elongata ${ }^{4}$ \\
\hline Haemophilus parainfluenzae (1) & S; SI & Actinobacillus hominis \\
\hline Haemophilus parainfluenzae ${ }^{5}(1)$ & S; SI & Aggregatibacter aphrophilus ${ }^{4}$ \\
\hline Haemophilus parainfluenzae (1) & S; SI & Pasteurella multocida ${ }^{6}$ \\
\hline Kingella denitrificans (2) & S; SC & K. denitrificans ${ }^{4}$ \\
\hline Kingella denitrificans (2) & S; SI & Neisseria bacilliformis \\
\hline Moraxella catarrhalis (1) & $\mathrm{S} ; \mathrm{SI}$ & M. nonliquefaciens \\
\hline Moraxella catarrhalis (2) & S; SI & M. osloensis \\
\hline Moraxella catarrhalis (1) & $\mathrm{S} ; \mathrm{SI}$ & Neisseria elongata ${ }^{4}$ \\
\hline Neisseria cinerea (1) & S; SC & N. cinerea ${ }^{4}$ \\
\hline Neisseria elongata (1) & S; SI & Capnocytophaga canimorsus ${ }^{4}$ \\
\hline Neisseria elongata (1) & $\mathrm{S} ; \mathrm{SI}$ & Capnocytophaga gingivalis ${ }^{4}$ \\
\hline Neisseria elongata (1) & $S ; S I$ & Eikenella corrodens ${ }^{4}$ \\
\hline Neisseria elongata (3) & S; SC & N. elongata ${ }^{4}$ \\
\hline Neisseria elongata (4) & $S ; S I$ & N. weaveri \\
\hline Neisseria gonorrhoeae (1) & $\mathrm{S} ; \mathrm{SI}$ & Moraxella lacunata \\
\hline Neisseria sicca (1) & S; SC & N. sicca ${ }^{4}$ \\
\hline Neisseria sicca (2) & $\mathrm{S} ; \mathrm{SI}$ & N. subflava \\
\hline Neisseria elongata (1) & $\mathrm{S} ; \mathrm{SI}$ & N. zoodegmatis \\
\hline Suttonella indologenes (1) & S; SI & $\begin{array}{l}\text { Aggregatibacter } \\
\text { actinomycetemcomitans }{ }^{4}\end{array}$ \\
\hline Not identified (1) & $\mathrm{N}$ & Aggregatibacter aphrophilus ${ }^{4}$ \\
\hline Not identified (1) & $\mathrm{N}$ & Moraxella atlantae \\
\hline Not identified (1) & $\mathrm{N}$ & Moraxella canis \\
\hline Not identified (3) & $\mathrm{N}$ & Moraxella nonliquefaciens \\
\hline Not identified (2) & $\mathrm{N}$ & Moraxella osloensis \\
\hline Not identified (1) & N & Neisseria animaloris \\
\hline Not identified (3) & N & Neisseria elongata ${ }^{4}$ \\
\hline Not identified (1) & $\mathrm{N}$ & Neisseria zoodegmatis \\
\hline Not identified (2) & N & Pasteurella bettyae \\
\hline Not identified (5) & $\mathrm{N}$ & Pasteurella multocida ${ }^{6}$ \\
\hline Not identified (1) & $\mathrm{N}$ & Pasteurella stomatis \\
\hline
\end{tabular}

${ }^{1}$ Final identification was assigned using 16S rRNA gene identification as the reference method and if required with supplemental conventional tests.

${ }^{2}$ Assignment to taxonomic level: $\mathrm{S}=$ species, $\mathrm{G}=$ genus, $\mathrm{N}=$ not identified.

${ }^{3}$ Correctness of assignment: SC = correct at species level, $\mathrm{SI}=$ incorrect at species level, $\mathrm{GC}=$ correct at genus level, $\mathrm{Gl}=$ incorrect at genus level, $\mathrm{N}=$ not identified .

${ }^{4}$ Taxon included in the VITEK 2 NH database; Capnocytophaga spp. is included as genus.

${ }^{5}$ Accepted as correct genus as Haemophilus aphrophilus was renamed as Aggregatibacter aphrophilus [22].

${ }^{6}$ Pasteurella multocida is included in the database of the VITEK 2 ID GNB card (bioMérieux). 


\section{Discussion}

In this study, we analysed a large set of fastidious GNR clinical isolates covering diverse genera and species, which were obtained under routine conditions in a diagnostic microbiology laboratory. Molecular identification is vastly superior to conventional identification, both in number of isolates assigned to correct taxon level and in accuracy (Table 2). A minority (6\%) of the 158 isolates included in the study could not be assigned to species level by $16 \mathrm{~S}$ rRNA gene sequence analysis. In contrast, $47 \%$ of the 158 isolates were not identified or misidentified by conventional phenotypic methods (Table 2). However, the performance of supplemental phenotypic tests was helpful to support the molecular identification in cases with low demarcation of two or more species due to highly similar $16 \mathrm{~S}$ rRNA gene sequences (Table 1).

Although the overall correct assignment to taxa by conventional phenotypic methods was rather poor, some species are easily assigned to correct species level by conventional identification procedures (Table 3 ). These are $A$. aphrophilus, C. hominis, E. corrodens, P. multocida and Capnocytophaga sp. other than C. canimorsus, which are characterised by typical biochemical key reactions that readily differentiate them from other fastidious GNR. In contrast, genera of Moraxella and Neisseria represent a challenge for the biochemical identification. Both genera often show similar biochemical reaction patterns, e.g., positive oxidase reaction or missing acid production from glucose, sucrose, maltose, mannitol, and xylose in semisolid cystine-trypticase agar medium; furthermore, the morphology in the Gram-stain does often not differentiate Moraxella and Neisseria species [13].

As alternative to conventional phenotypic methods, we analysed a subgroup of 80 isolates of fastidious GNR by the commercially available colorimetric VITEK $2 \mathrm{NH}$ card (bioMérieux). Despite the limited database, this system supports the identification of fastidious GNR similar to that of conventional biochemical reactions by identifying $31 \%$ and $9 \%$ of the isolates to correct species and genus level, respectively.

Accurate identification of clinically relevant isolates of fastidious GNR is important for adequate interpretation and reporting as infectious agents and susceptibility testing [1]. However, in a routine diagnostic microbiology laboratory it is not feasible to subject all clinical isolates to molecular analyses for identification. Mahlen et al. proposed an efficient strategy by applying selective criteria such as discordant morphologic or biochemical results and knowledge of validity of phenotypic testing of isolates of Gram-negative bacilli [23]. Based on our data, we propose a cost-efficient algorithm, which is based on the knowledge of easy-to-identify organisms by conventional phenotypic methods and molecular analyses by the 16S rRNA gene for other difficult-to-differentiate species of this group. For identification of fastidious GNR conventional biochemical reactions and 16S rRNA gene sequence analysis can be implemented in a diagnostic laboratory as follows: (i) conventional biochemical identification of A. aphrophilus, C. hominis, E. corrodens, and $P$. multocida based on the typical reaction pattern is reliable; and (ii) any other result including Capnocytophaga sp. should be subjected to molecular methods by $16 \mathrm{~S}$ rRNA gene analysis when accurate identification is of concern. By applying this approach to the 158 fastidious GNR analysed in our study, at least a third (32\%) of the isolates would be readily identified by conventional phenotypic methods without laborious molecular analyses.

\section{Conclusions}

In time of cost-effectiveness and rapid development of newer identification methods such as MALDI-TOF MS, an efficient strategy for difficult-to-identify bacteria is mandatory as alternative method. In this study we analysed a substantial set of various clinical isolates covering 20 genera and 50 species of fastidious GNR and evaluated the reliability of both conventional phenotypic methods and 16S rRNA gene analyses for accurate identification of such microorganisms. We propose an identification algorithm for fastidious GNR for a routine diagnostic laboratory as follows: (i) conventional biochemical identification of $A$. aphrophilus, C. hominis, $E$. corrodens, and P. multocida based on the typical reaction pattern is reliable; and (ii) any other result including Capnocytophaga sp. should be subjected to molecular methods by $16 \mathrm{~S}$ rRNA gene analysis when accurate identification is of concern.

\section{Competing interests}

The authors declare that they have no competing interests.

\section{Authors' contributions}

$\mathrm{MMO}$ contributed to the acquisition of laboratory data, analysis of biochemical data and drafting the manuscript. SA contributed to the overall study design and acquisition of molecular data. GVB contributed to the overall study design and critical revision of the draft. RZ contributed to the overall study design, analysis and interpretation of biochemical data and helped to draft the manuscript. AZ contributed to the acquisition of laboratory data, molecular analyses, evaluation of the sequence data and drafting the manuscript. All authors read and approved the final manuscript.

\section{Acknowledgements}

This study was supported in part by the University of Zurich. The authors thank F. Gürdere, J. Giger and the laboratory technicians for their dedicated help. We thank E. C. Böttger for continuous support and critical reading of the manuscript.

\section{Author details}

${ }^{1}$ Institute of Medical Microbiology, University of Zurich, 8006 Zurich, Switzerland. ${ }^{2}$ Present address, Policlínica Nascente, Prefeitura Municipal de Fortaleza, Fortalez-Ceará, Brasil. ${ }^{3}$ Present address, Institute for Hygiene and Public Health, University Hospital, 53105 Bonn, Germany.

Received: 5 January 2013 Accepted: 12 July 2013

Published: 16 July 2013 


\section{References}

1. Zbinden R, von Graevenitz A: Actinobacillus, Capnocytophaga, Eikenella, Kingella, Pasteurella, and other fastidious or rarely encountered Gramnegative rods. In Manual of Clinical Microbiology. Volume 1. 10th edition. Edited by Versalovic J, Carroll KC, Funke G, Jorgensen JH, Landry ML, Warnock DW. Washington DC: ASM press; 2011:574-588.

2. Brouqui $P$, Raoult D: Endocarditis due to rare and fastidious bacteria. Clin Microbiol Rev 2001, 14(1):177-207.

3. Tang YW, Ellis NM, Hopkins MK, Smith DH, Dodge DE, Persing DH: Comparison of phenotypic and genotypic techniques for identification of unusual aerobic pathogenic gram-negative bacilli. $J$ Clin Microbiol 1998, 36(12):3674-3679.

4. Rennie RP, Brosnikoff C, Shokoples S, Reller LB, Mirrett S, Janda W, Ristow K, Krilcich A: Multicenter evaluation of the new Vitek 2 NeisseriaHaemophilus identification card. J Clin Microbiol 2008, 46(8):2681-2685.

5. Sonksen UW, Christensen JJ, Nielsen L, Hesselbjerg A, Hansen DS, Bruun B: Fastidious Gram-negatives: identification by the Vitek 2 NeisseriaHaemophilus card and by partial 16S rRNA gene sequencing analysis. Open Microbiol J 2010, 4:123-131.

6. Valenza G, Ruoff C, Vogel U, Frosch M, Abele-Horn M: Microbiological evaluation of the new Vitek 2 Neisseria-Haemophilus identification card. J Clin Microbiol 2007, 45(11):3493-3497.

7. Couturier MR, Mehinovic E, Croft AC, Fisher MA: Identification of HACEK clinical isolates by matrix-assisted laser desorption ionization-time of flight mass spectrometry. J Clin Microbiol 2011, 49(3):1104-1106.

8. Tan KE, Ellis BC, Lee R, Stamper PD, Zhang SX, Carroll KC: Prospective evaluation of a matrix-assisted laser desorption ionization-time of flight mass spectrometry system in a hospital clinical microbiology laboratory for identification of bacteria and yeasts: a bench-by-bench study for assessing the impact on time to identification and cost-effectiveness. J Clin Microbiol 2012, 50(10):3301-3308.

9. van Veen SQ, Claas EC, Kuijper EJ: High-throughput identification of bacteria and yeast by matrix-assisted laser desorption ionization-time of flight mass spectrometry in conventional medical microbiology laboratories. J Clin Microbiol 2010, 48(3):900-907.

10. Clarridge JE: Impact of $16 \mathrm{~S}$ rRNA gene sequence analysis for identification of bacteria on clinical microbiology and infectious diseases. Clin Microbiol Rev 2004, 17(4):840-862.

11. Woo PC, Lau SK, Teng JL, Tse H, Yuen KY: Then and now: Use of 165 rDNA gene sequencing for bacterial identification and discovery of novel bacteria in clinical microbiology laboratories. Clin Microbiol Infect 2008, 14(10):908-934.

12. von Graevenitz A, Funke G: An identification scheme for rapidly and aerobically growing gram-positive rods. Zentralbl Bakteriol 1996, 284(2-3):246-254.

13. Weyant RS, Moss CW, Weaver RE, Hollis DG, Jordan JG, Cook EC, Daneshvar MI: Identification of unusual pathogenic Gram-negative aerobic and facultatively anaerobic bacteria. 2nd edition. Baltimore: Williams \& Wilkins; 1996.

14. Bosshard PP, Abels S, Altwegg M, Böttger EC, Zbinden R: Comparison of conventional and molecular methods for identification of aerobic catalase-negative Gram-positive cocci in the clinical laboratory. J Clin Microbiol 2004, 42(5):2065-2073.

15. Bosshard PP, Abels S, Zbinden R, Böttger EC, Altwegg M: Ribosomal DNA sequencing for identification of aerobic Gram-positive rods in the clinical laboratory (an 18-month evaluation). J Clin Microbiol 2003, 41(9):4134-4140

16. Bosshard PP, Zbinden R, Abels S, Böddinghaus B, Altwegg M, Böttger EC 16S rRNA gene sequencing versus the API 20 NE system and the Vitek 2 ID-GNB card for identification of nonfermenting Gram-negative bacteria in the clinical laboratory. J Clin Microbiol 2006, 44(4):1359-1366.

17. CLSI: Interpretive criteria for identification of bacteria and fungi by DNA target sequencing; approved guideline (MM18-A). Wayne, PA: Clinical and Laboratory Standards Institute; 2008

18. Elias J, Frosch M, Vogel U: Neisseria. In Manual of Clinical Microbiology. Volume 1. 10th edition. Edited by Versalovic J, Carroll KC, Funke G, Jorgensen JH, Landry ML, Warnock DW. Washington DC: ASM press; 2011:559-573.

19. Kämpfer $P$, Vaneechoutte $M$, Lodders $N$, De Baere T, Avesani V, Janssens $M$, Busse HJ, Wauters G: Description of Chryseobacterium anthropi sp. nov. to accommodate clinical isolates biochemically similar to Kaistella koreensis and Chryseobacterium haifense, proposal to reclassify Kaistella koreensis as Chryseobacterium koreense comb. nov. and emended description of the genus Chryseobacterium. Int I Syst Evol Microbiol 2009, 59:2421-2428.

20. Vaneechoutte M, Dijkshoorn L, Nemec A, Kämpfer P, Wauters G: Acinetobacter, Chryseobacterium, Moraxella, and other nonfermentative Gram-negative rods. In Manual of Clinical Microbiology. Volume 1. 10th edition. Edited by Versalovic J, Carroll KC, Funke G, Jorgensen JH, Landry ML, Warnock DW. Washington DC: ASM press; 2011:714-738.

21. Zbinden R: Capnocytophaga canimorsus: challenge for the clinical microbiologist. Med Microbiol Lett 1995, 4:217-223.

22. Norskov-Lauritsen N, Kilian M: Reclassification of Actinobacillus actinomycetemcomitans, Haemophilus aphrophilus, Haemophilus paraphrophilus and Haemophilus segnis as Aggregatibacter actinomycetemcomitans gen. nov., comb. nov., Aggregatibacter aphrophilus comb. nov. and Aggregatibacter segnis comb. nov., and emended description of Aggregatibacter aphrophilus to include V factordependent and V factor-independent isolates. Int I Syst Evol Microbiol 2006, 56:2135-2146.

23. Mahlen SD, Clarridge JE: Evaluation of a selection strategy before use of 16S rRNA gene sequencing for the identification of clinically significant Gram-negative rods and coccobacilli. Am J Clin Pathol 2011, 136:381-388.

doi:10.1186/1471-2180-13-162

Cite this article as: de Melo Oliveira et al:: Accurate identification of fastidious Gram-negative rods: integration of both conventional phenotypic methods and 16S rRNA gene analysis. BMC Microbiology 2013 13:162.

\section{Submit your next manuscript to BioMed Central and take full advantage of:}

- Convenient online submission

- Thorough peer review

- No space constraints or color figure charges

- Immediate publication on acceptance

- Inclusion in PubMed, CAS, Scopus and Google Scholar

- Research which is freely available for redistribution 